\title{
THE REALISM OF STOCHASTIC WEATHER GENERATORS IN RISK DISCOVERY
}

\author{
ABDULLAH ALODAH ${ }^{1,2}$ \& OUSMANE SEIDOU ${ }^{1}$ \\ ${ }^{1}$ Department of Civil Engineering, University of Ottawa, Canada \\ ${ }^{2}$ Department of Civil Engineering, Qassim University, Saudi Arabia
}

\begin{abstract}
Weather generators reproduce artificial climate time series that are commonly used for hydrological modeling and climate adaptation studies. To examine the representativeness of a stochastically generated climate time series, a novel stochastic method is suggested where these time series are projected in two spaces (the Climate Statistics Space - CSS; and the Risk and Performance Indicators Space - RPIS). A visual inspection as well as the Mahalanobis distance are used to assess the two spaces relative position and their proximity to the points representing the observations. The dimensions of the CSS are a subset of climate statistics, while the dimensions of the RPIS are a set of risk and performance indicators calculated using streamflow time series. A rainfall-runoff model is used to convert all climate time series from the CSS into streamflow time series in the RPIS. Three stochastic weather generators were used in this study: The Weather Generator École de Technologie Supérieure (WeaGETS), the Multisite Stochastic Weather Generator (MulGETS) using two different generation algorithms, and a k-nearest neighbour weather generator. Each generator was used to construct precipitation, maximum and minimum temperatures time series representing the historical period. The suggested approach was tested on a 41-years-long climate and flow time series from South Nation watershed in Eastern Ontario, Canada. The MulGETS model was able to perform well where the point representing the observations was centered inside the cloud of points representing the synthetic time series in some CSS.

Keywords: stochastic hydrology, hydrological modeling, weather generators assessment, risk and performance indicators.
\end{abstract}

\section{INTRODUCTION}

In climate change studies, stochastic weather generators are sometimes used to evaluate the risk and performance profile of water resources systems. They are used to generate highresolution synthetic climate sequences that are used in many hydrological applications to calculate streamflow rates and to evaluate alternative designs and policies [1], [2]. Stochastic weather generators attempt to generate daily time series of atmospheric variables having the statistical characteristics of historical climate data. Weather generators typically used as inputs into more complex hydrological, environmental, ecological or agricultural impact studies. Despite the fact that several approaches and techniques have become more sophisticated, the accuracy of reproduced climates has not always been adequately justified.

Historically, since the eighties of the past century, a number of weather generators (WGs) has been put forward to serve the climate, agricultural and hydrological aspects and more recently in climate change impact assessment (for example, Forsythe et al. [3]). Fowler et al. [4] criticized WGs for poor modelling of inter-annual variability in monthly means and for being strictly localized; thus, they may not be useful in other climates. In contrast, Wilby and Fowler [5] indicated that WGs could be practical and useful for some applications, such as the count of wet spells and monthly precipitation for hydrological applications in developing countries.

While a generally accepted approach to evaluate WGs in hydrology field is lacking, the current research is proposing a stochastic technique to examine WGs. The framework proposed by Brown et al. [6] was adopted in which a climate state represented by a time 
series $X_{t}$ is summarized by a subset of climate statistics $\mathcal{V}_{\mathrm{T}}$ calculated over period $\mathrm{T}(\mathrm{T}=41$ years in observed and synthetic data) that are relevant to the problem under investigation (e.g., the mean standard variation of precipitation and temperature). $\mathcal{V}_{\mathrm{T}}$ is calculated for observations time series and stochastically generated time series. A climate state will yield a level of risk and performance that is measured by a set of risk/performance indicators $\mathcal{V}_{T}$ is obtained by feeding an impact model with time series $\mathrm{X}_{\mathrm{t}}$. This leads to all time series being represented by a point in one of two spaces called the Climate Statistics Space (CSS) and the Risk and Performance Indicators Space (RPIS). The dimensions of the CSS are a subset of climate statistics (e.g., mean, standard deviation), while the dimensions of the RPIS are a set of risk and performance indicators calculated using streamflow time series (e.g., flood risk, generated hydropower). Each climate time series, either observed or generated is projected in the CSS. ASWAT (Soil and Water Assessment Tool) rainfall runoff model is used to convert climatic time series (either observed or generated) into streamflow time series. The streamflow time series (either observed or generated) are projected in the RPIS space. A visual inspection as well as the Mahalanobis distance is used to assess their relative position and their proximity to the points representing the observations, and rank the performance of competing weather generators. The current research is an attempt to generate criteria that can be used to assess some available weather generators on the test case and developing a recommendation on which ones should be used for risk discovery.

\section{STUDY AREA, CLIMATE DATA AND MATERIALS}

\subsection{Study area}

The study area is the South Nation watershed located in Eastern Ontario, Canada, spanning between $75^{\circ} 43^{\prime} \mathrm{W}-74^{\circ} 22^{\prime} \mathrm{W}$ longitude and $44^{\circ} 40^{\prime} \mathrm{N}-45^{\circ} 38^{\prime} \mathrm{N}$ latitude as shown in Fig. 1. Its drainage area is approximately $4000 \mathrm{~km}^{2}$ used mostly for agriculture. The area is drained by the South Nation River that runs northeast from Brockville for $175 \mathrm{~km}$ towards Plantagenet. The South Nation watershed is relatively flat where the river has a low topographic gradient of $80 \mathrm{~m}$ between its headwaters and the confluence with the Ottawa River. Hence, The South Nation watershed is poorly drained which consequently maximizes the flooding risk and boosts the erosion of riverbanks and of topsoil on agricultural lands.

\subsection{Climate data}

Meteorological data were obtained from four gauges over a period of 41 years (Jan. 1971Dec. 2011) to represent the South Nation watershed as shown in Fig. 1. These stations include St. Albert, Russell, Morrisburg and South Mountains. The area, in average, received approximately $985 \mathrm{~mm}$ of precipitation annually and its annual mean maximum and minimum temperatures are 11.5 and 1.2 degrees Celsius, respectively (Environment Canada [7]).

\subsection{Weather generators}

\subsubsection{Comparison of WGs}

In general, weather generators are designed to deal with records of a single station [8]. Such models are not powerful to capture the spatial attributes of the watershed stations by producing time series for each station independently. Alternatively, multi-site based generators have been introduced to fill such need. Baigorria and Jones [9] classify multi-site weather generators based on their structure and mathematical algorithms into three 


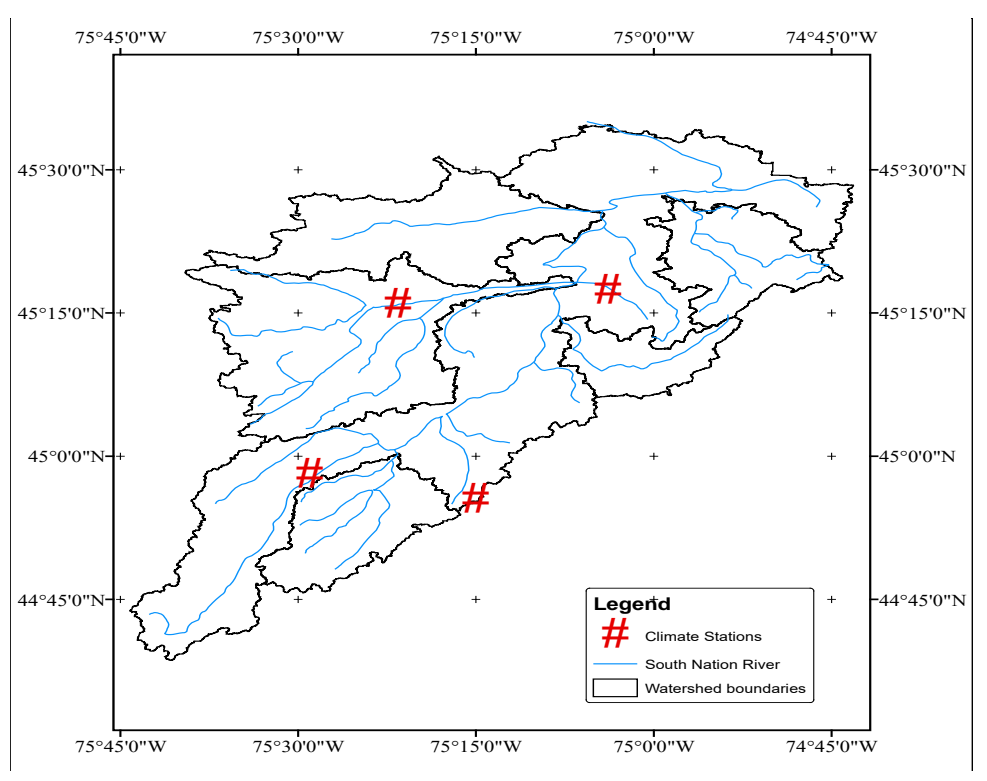

Figure 1: The map of the South Nation watershed.

classifications: parametric (such as Wilks [10]), non- parametric (such as Wilby et al. [11], and hybrid (such as Fowler et al. [4], Palutikof et al. [12]). A multi-site weather generator is recommended (Chen et al. [13]) for relatively large watersheds, whereas stations of small watersheds can be integrated into single station using some graphical techniques such as Thiessen Polygon or by simply using a sole representative station.

It is highly recommended to make a multi-comparison study of variety of available weather generators that apply different methodologies and techniques. The suggested approach was conducted on three weather generators: MulGETS, WeaGETS and k-nearest neighbour resampling approaches.

A considerable amount of literature has been published on the best-fit probability distribution function of precipitation. The gamma distribution function has been intensely preferred to wet-day daily precipitation (e.g., Thom [14]; Buishand [15]; Geng et al. [16]; Sen and Eljadid, [17]). Moreover, other distributions have been recommended such as exponential (e.g., Woolhiser and Roldan [18], Wilks [10]), Weibull (e.g. Burgueño et al. [19]) and the Pearson Type-III (P3) distribution to be applied to the full record of daily precipitation data (Hanson and Vogel [20]).

\subsubsection{WeaGETS and MulGETS}

The Matlab-based WeaGETS (Chen et al. [21]) is an extension to WGEN model (Richardson and Wright [22]) and provides three orders of Markov chains to precisely account for wet and dry spells and a smoothing scheme to modify the precipitation parameters for generating precipitation occurrence. For precipitation quantities, two distributions are provided: Gamma (WG) and Exponential (WE). It uses a first-order linear autoregressive model to synthetically generate maximum and minimum temperatures. WeaGETS is suitable for small watersheds where a single station can be used to represent the entire watershed [21]. In spite of that, there is a need to use multi-site WG for large basins. Mehrotra et al. [23] indicated that Multi-site 
weather generators are preferable in many applications and that hydrological simulations require precipitation data to be spatially correlated. Thus, the new version introduced by Chen et al. [13] provided a weather generator that take into account the spatial attributes of the climate data. Chen et al. [21] developed a multisite, multivariate weather generator so-called the Multi-site weather Generator of École de Technologie Supérieure (MulGETS) to simulate daily precipitation and temperature. The same model used in its counterparts WeaGETS is applied to reproduce precipitation and temperature in MulGETS. It follows the precipitation algorithm as described in detail in Brissette et al. [24]. However, MulGETS constructs random values that are spatially correlated but temporally independent [21]. MulGETS offers the option of using a multi-gamma distribution (a combination of several gamma distributions), and a multi- exponential distribution, to simulate daily precipitation amounts.

Precipitation sequences were being produced in the MulGETS and WeaGETS models using two different distributions: one-parameter exponential and two-parameter gamma. The probability density function ( $\mathrm{pdf}$ ) of exponential distribution is:

$$
f(x)=\lambda e-\lambda x,
$$

where $\mathrm{x}$ is the daily precipitation amount and its parameter $\lambda$ equals $1 /$ mean. The multiexponential distribution combines several exponential distributions, which each has its own parameter. The probability density distribution (pdf) of gamma is given by:

$$
f(x)=\frac{(x / \beta)^{\alpha-1} \exp [-x / \beta]}{\beta \Gamma(\alpha)},
$$

where $\alpha$ and $\beta$ are the shape and scale parameters respectively, and $\Gamma(\alpha)$ denotes the gamma function calculated at $\alpha$. The two parameters $(\alpha$ and $\beta)$ required in order to use the gamma distribution are directly linked to the mean $(\mu)$ and the standard deviation $(\sigma)$. They are defined as follows:

$$
\begin{gathered}
\mu=\alpha / \beta \\
\sigma=\sqrt{\alpha} / \beta
\end{gathered}
$$

Chen et al. [21] indicated that exponential distribution is easier to calculate but has less performance compared to the gamma distribution. Hence, gamma distribution is commonly utilized to generate daily precipitation quantity.

\subsubsection{K-nearest neighbour}

Two approaches that presumably are capable of generating realistic precipitation and temperature sequences were implemented in the analysis. Precipitation sequences were generated applying the model initiated by Goyal et al. [25] that uses the k-nearest neighbor method coupled with gamma kernel perturbation. The later seemingly allows producing values that are not covered in the historical data. For maximum and minimum temperatures, with no underlying probability distribution assumptions, Sharif and Burn [26] method was implemented to generate temperature sequences.

\subsection{The hydrological model SWAT}

The Soil and Water Assessment Tool (SWAT) is a semi-distributed, watershed-scale hydrologic model used broadly to address water quality and quantity issues since established in the early 1990s [27]. Hydrologists, conservationists and policy makers have extensively used SWAT to predict such issues and their environmental impacts (e.g., Neitsch et al. [27], Srinivasan and Arnold [28], White and Chaubey [29], Tuppad et al. [30], Arnold et al. [31]). 
The main physically based inputs that govern transformation of precipitation to runoff in SWAT are weather information [31]. SWAT's comprehensive physical-process parameterizations strengthen its capability to provide sufficient information for adequate watershed management decisions. To deal with spatial variations in a watershed of interest, a watershed is divided into a number of hydrologic response units (HRUs) within sub-basins. Beside land cover and soil type, each HRU has its own features such as weather, groundwater, plant growth, land-use, and soil type [27]. A total of 31 sub-basins were constituted in this watershed to adequately simulate aerial distribution of hydrological processes. Precipitation and temperature data of the four selected stations representing the watershed beside streamflow data were used.

By assuming, initially, a reasonably large parameter uncertainty, the SUFI-2 (sequential uncertainty fitting ver. 2) optimization algorithm that follows an inverse modelling procedure (Abbaspour et al. [32]) was implemented for parameterizations to capture the narrowest prediction uncertainty range. The optimal goal is to maximize P-factor to $100 \%(0.63)$ and minimize D-factor to zero (for detailed explanation see Abbaspour et al. [32]). While 25 parameters were implemented, a sample of 5 parameters bounds beside the best fitted values within prediction uncertainty band are provided in Table 2 .

\section{METHODOLOGY}

\subsection{Assessing the adequacy of a given stochastic weather generator}

SWAT, after being calibrated with observed climate data, is driven with a set of weather synthetic time series of precipitation, maximum and minimum temperatures generated by the WG under investigation to obtain time series of streamflow that are afterward projected in the RPIS along with observed streamflow. The relative position of the points representing each time series in the RPIS allows. A good WG would generate points that symmetrically spread around the point representing observations; the spread of the points on each dimension should represent the variability of the risk indices being calculated given the length of the time series and the natural variability of historical streamflow data.

In the proposed approach, one thousand samples were represented by their statistics $\left(\mathcal{V}_{\mathrm{T}}\right)$ in the confidant interval figures. The objective is to discover how rarely an observation event to occur in the cluster of experiments. In other words, the observation event is tested against the normal pattern of weather generators samples using an adoptive threshold.

The realism of a stochastically generated climate time series can be assessed using the following two criteria: (a) checking if the point representing the observations is centered inside the cloud of points representing the synthetic time series in the CSS and the RPIS and

Table 1: Description of some SWAT2012 input parameters.

\begin{tabular}{|c|c|c|c|c|}
\hline \multirow{2}{*}{ Parameter name* } & \multirow{2}{*}{ Description } & \multicolumn{2}{|c|}{ Initial range } & \multirow{2}{*}{$\begin{array}{l}\text { Fitted } \\
\text { values }\end{array}$} \\
\hline & & Min & $\operatorname{Max}$ & \\
\hline $\mathrm{r} \_\mathrm{CN} 2 . \mathrm{mgt}$ & Curve number for moisture condition II & -0.2 & 0.2 & -0.06 \\
\hline V_ALPHA_BF.gw & Baseflow alpha factor & 0.1 & 0.9 & 0.70 \\
\hline v_GW_DELAY.gw & Groundwater delay time & 1 & 499 & 206.65 \\
\hline v_GWQ̄MN.gw & $\begin{array}{l}\text { Threshold depth of water in the shallow } \\
\text { aquifer required for return flow to occur } \\
\left(\mathrm{mm} \mathrm{H}_{2} \mathrm{O}\right) \text {. }\end{array}$ & 1 & 4900 & 2499.05 \\
\hline $\mathrm{v}$ CH K2.rte & Main channel conductivity & -0.09 & 400 & 40.89 \\
\hline
\end{tabular}


(b) checking if proximity with observations in the CSS translates into proximity in the risk/performance indicators space. Failure to meet criterion (a) would mean that the weather generator is biased toward one zone of the CSS. Failure to meet criterion (b) would mean that some important statistical characteristics have been overlooked and are not being reproduced by the weather generator.

The streamflow attributes or measures of central tendency were used to examine whether a weather generator reproduces the measured streamflow distributions well in a monthly temporal scale. By examining the distributions of mean against key attributes of datasets including variation (standard deviation), skewness $\left(\alpha_{3}\right)$ and kurtosis $\left(\alpha_{4}\right)$ of observed and stochastically produced climate, atmospheric variables should be reproduced correctly in a distributional sense.

\subsection{Multivariate Gaussian distribution}

Instead of relying on a single estimate, reliability interval estimates are alternatively used where ranges of values are reported. Such reliability estimates of a large enough sample set then tested against a pre-defined observed value. By running a large enough sample set of experiments (i.e. one thousand), a multivariate Gaussian distribution model can be built for the summarizing subset of climate statistics. This comprises working out the statistic features for each feature of these experiments. Then, based upon a minimum membership probability, a new sample (i.e. observations in this case) is tested to check its qualification as a 'normal' sample within the examined weather generator. The multivariate Gaussian distribution is computed as:

$$
p(x)=\frac{1}{(2 \pi)^{d / 2}|\Sigma|^{1 / 2}} \exp \left[-\frac{1}{2}(x-\mu)^{T} \Sigma^{-1}(x-\mu)\right],
$$

where the mean, $\mu$, is a d length row vector and the covariance, $\Sigma$, is a d by $\mathrm{d}$ matrix.

A cloud or cluster is formed to represent normally distributed where the mean and covariance matrix determine the position and the shape of the cloud.

\subsubsection{Mahalanobis distance}

Mahalanobis distance, called also the generalized squared distances, is a tool frequently used in the detection of anomalous, or for discriminant analysis indicating the probability of a certain sample, of belonging to a certain group (Huber and Ronchetti [33], Fritsch et al. [34], Wang and Zwilling [35]). Mahalanobis distance (MD) is a quadratic distance from a preselected point $x_{i} \in \mathbb{R}^{\mathrm{P}}$ (an event representing observations) to a location $\mu$ (a centre of a cloud) governed by a covariance matrix $\Sigma$ (a shape parameter) that is given by the quantity:

$$
d_{m}^{2}\left(x_{i}, \mu\right)=\left(x_{i}-\mu\right)^{T} \Sigma^{-1}\left(x_{i}-\mu\right)
$$

Moreover, Mahalanobis distance calculates the distance between two points in a space and is denoted by MD.

The set of points that satisfied or determined by invariant Mahalanobis distances will form an ellipse (bivariate) or ellipsoid (multivariate). The principal axes of an ellipse and their lengths are governed by the eigenvectors $(\Phi)$ of the covariance vector $(\Sigma)$, and the eigenvalues $(\Lambda)$ respectively. The smaller MD value the closer a sample to be positioned on or close to the mean values (i.e. the focus) of the ellipse and further points in contrast will have higher values and consequently more likely to be outliers. However, it is important to bear in mind the possibility, especially for high dimensional multivariate, that an ordinary sample could be detected as an outlier if one attribute is considerably biased. Yet, defining a particular threshold for outlying distances should be done with some cautious as it depends 
on the application and the type of samples. In theory, the Mahalanobis squared distance describes how deviated a point from the group mean in units of standard deviation, thus, about $68 \%, 95 \%, 99.7 \%$ of data are circumscribed within 1, 2 and 3 standard deviations around the mean, respectively. Hence, a point of Mahalanobis squared distance of 3 or less lies within the boundary of $99 \%$ of data. It is well documented that the MD is described by a chi-square $\left(\chi^{2}\right)$ distribution with a degree of freedom (df) equivalent to the number of independent variables (Hardin and Rocke [36]). A tested weather generator is called a goodfit candidate when the reference point of observations (represented by its features) falls within a reasonable distance from the investigated data center. Observed data were examined as a sample unit to the group of sample units of a weather generator. Assessed with a very conservative probability of $\mathrm{p}<0.01$, a critical value of chi-square $(\alpha)$ with degrees of freedom equal to the number of variables (i.e. $\mathrm{df}=2$ ) is 13.82 .

\section{RESULTS, DISCUSSION AND CONCLUSION}

Based on Liew et al. [37] and Moriasi et al. [38] criterion for discharge data at a monthly temporal scale, values of NSE, RSR and PBIAS were found to be $0.792,0.45$ and $3 \%$ respectively, which indicate a very good model.

Mean, standard deviation, skewness and kurtosis of annual Tmax, Tmin and precipitation samples generated synthetically were compared to observed ones. Similarly, mean, standard deviation, skewness and kurtosis of monthly streamflow simulated by SWAT and fed with synthetic climate were compared to observed discharge. Although sitting a clear boundary between normal and anomalous behaviors can be controversial, anomaly detection methods are applied to examine whether the historical event lies inside of a predefined set of normal behaviors. A weather generator must fit a set of criteria. The desired behavior of a training set of a tested weather generator is to surround the observed data in both spaces. Correspondingly, a weather generator is classified as a good fit for a specific location when MD is less than 3. In addition, the reference point is said to be an anomaly event when its probability to occur in certain space is very low. The shape of the distribution is determined by its skewness and kurtosis, which plays a crucial role in extreme values investigation. The mean, on the other side, is important to predict streamflow quantities and standard deviation explains the spread about the mean.

\subsection{Climate Statistics Space (CSS)}

The WeaGETS third-order Markov model, which increases the number of parameters, without parameter smoothing was selected to adequately generate long dry or wet spells. This selection is consistent with previous works (e.g. Wilks [10], Chen et al. [21], Lennartsson et al. [39], Allard et al. [40]). Chen et al. [21] stated that generating precipitation quantity using the gamma distribution performed reliably better than the exponential distribution. Yet, results in Fig. 2-a suggests that the two distributions were comparable in the climate space where $\alpha_{3}$ and $\alpha_{4}$ were better reserved by exponential family whereas $\sigma$ was reproduced better by gamma family. These findings are partially consistent with Wilks's work [10] as exponential favoured over gamma. Both models, however, were not sufficiently competent in the artificially generated temperatures.

The k-nearest neighbor approach performed relatively less efficiently, where all observed attributes of historical precipitation data were not reproduced as MDs of the three central moments were way above the threshold of three. However, despite its inadequacy in precipitation, k-nn performed outstandingly in reproducing synthetic temperatures sequences by preserving the statistical features of observed data. 
A possible explanation for the difference in the performances of the WG might be that be attributed to the underlying numerical algorithms and the accuracy of representing the complex land-ocean-atmosphere interactions [2]. This also could be a limitation in weather generators to produce more efficiently in certain regions or climates depending on the weather systems. Fowler et al. [4] criticized WGs for being strictly localized; thus, they may not be useful in any climate regime.

\subsection{Risk and Performance Indicators Space (RPIS)}

The main objective is to discover how rarely discharge observations to occur in the cluster of experiments resulted from feeding SWAT with synthetic data. In other words, the historical statistics are tested against the normal pattern of samples based on an adoptive threshold. Streamflow data generated in SWAT fed with weather generators time series were tested against those resulted from SWAT fed with observed climate as shown in Fig. 2(b). MulGETS-Gamma, for the study area, is appealing to be the best weather generator in preserving basic statistics of original streamflow with the observation points at Mahalanobis distances of $0.85,0.3$ and 0.32 for $\sigma, \alpha_{3}$ and $\alpha_{4}$, respectively. It is followed by MulGETSExponential while the WeaGETS models and the k-nn approach were not capable of maintaining the historical statistics and in particular the higher central moments $\left(\alpha_{3}\right.$ and $\left.\alpha_{4}\right)$.

In general, weather models that have verified better in the CSS against the observed data appeal to some extent to perform sufficiently in the RPIS. Overall, the gamma distribution employing MulGETS and WeaGETS to producing streamflow was better fit to observed data than exponential distribution. Consequently, more weight can be given in the pilot watershed to MulGETS, implementing Gamma, to reproduce precipitation as it was capable of preserving the central moments very well where MD of $\sigma, \alpha_{3}$ and $\alpha_{4}$ were $0.45,0.48$ and 1.33 , respectively. K-nn can be used to generate synthetic maximum and minmum temperature time series.

\section{CONCLUSION}

The adequacy of weather generators to derive risk profile using hydrological risk indicators is assessed. A calibrated SWAT hydrological model was fed with climatic time series generated stochastically. Beside k-nn approaches, the Matlab-based stochastic weather generators MulGETS and WeaGETS were utilized to generate maximum and minimum temperatures as well as precipitation intensities and occurrences implementing exponential and gamma distributions for the selected watershed. The current study has only examined five families of weather generators coupled with a hydrological model while involving more models would lead emphatically to definitive implications. Overall, results indicated that the multi-site stochastic weather generator (MulGETS) has the best performance in the climate statistics space (CSS) followed by its counterpart for single site (WeaGETS) then k-nearest neighbour. The leading cause of diversity in weather generators outputs is the influence of the underlying assumptions and computational processes. The historical statistics of streamflow were found to be falling outside the predefined set of normal behaviour of the WeaGETS models and the k-nn approach. However, the MulGETS models, gamma followed by exponential, should be considered as good candidates for risk discovery in the South Nation watershed. Finally, it is also important to say that there is no binary classification of WGs but a WG could be suitable for a specific field or space more than others and fails in other fields. In other words, the suggested approach aims to highlight the importance to synthesize the CSS and the RPIS rather than one or two statistic tests in CSS. The current work appeals to climate product users for a carefully pick of WG based on 
(a)

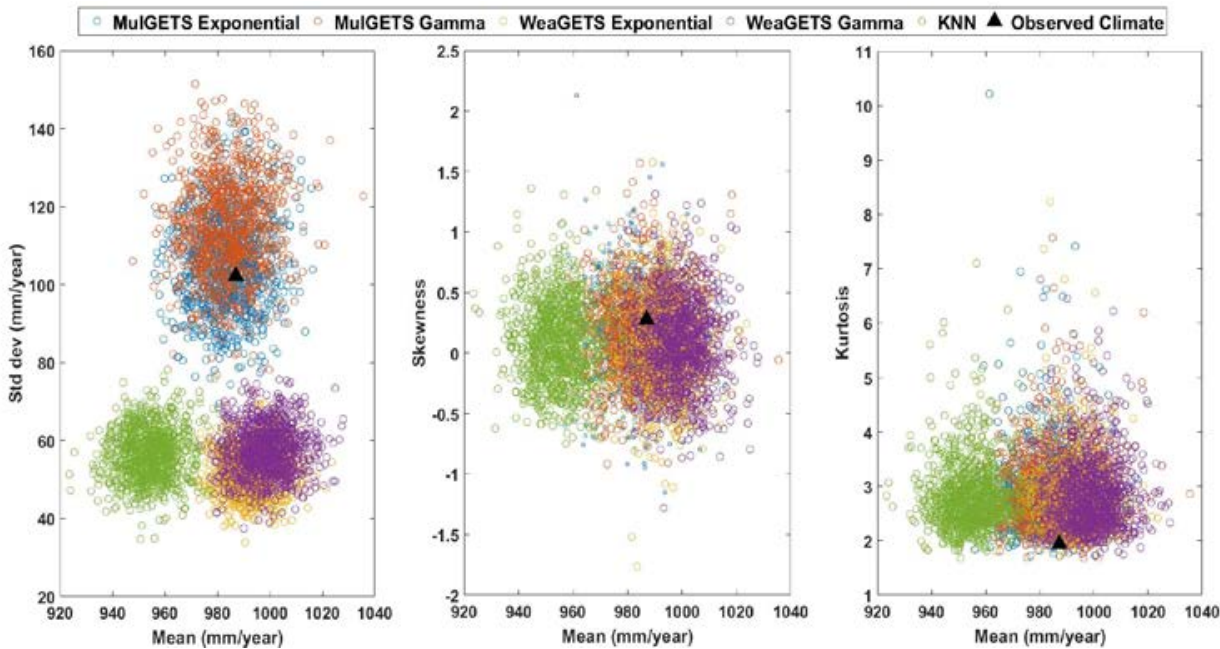

(b)

SWAT-MulGETS Exponential 0 SWAT-MuIGETS Gamma $\circ$ SWAT-WeaGETS Exponential 0 SWAT-WeaGETS Gamma SWAT-KNN $\boldsymbol{\Delta}$ sWAT-Observed Climate $\boldsymbol{\Delta}$ Observed Flow
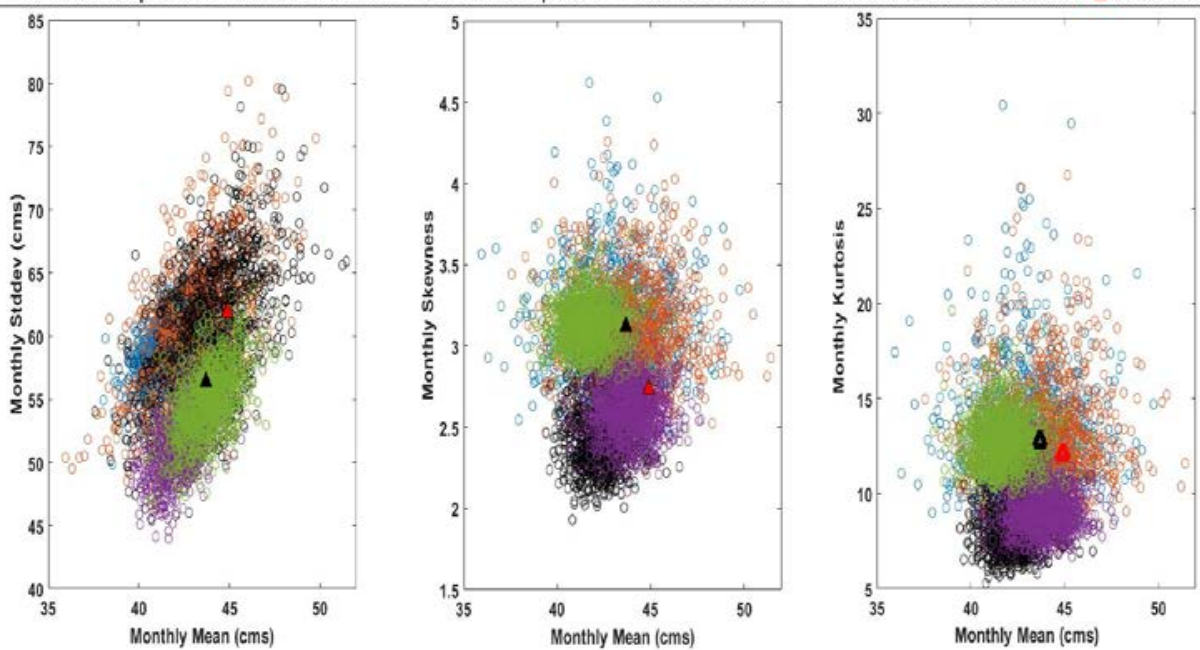

Figure 2: Scatter plots of statistics features of weather generators for: (a) Precipitation; and (b) Streamflow.

the relevant CSS and RPIS information. It is also worth looking to verify these results by versatile models such as economical, ecological, electricity demand or crop-yield models and to analyze their performance as well.

\section{REFERENCES}

[1] Loucks, D., Stedinger, J., \& Haith, D., Water Resource Systems Planning and Analysis, Prentice-Hall, Englewood Cliffs, NJ, 1981.

[2] Warner, T., Climate modeling and downscaling. Numerical Weather and Climate Prediction, pp. 407-455, Cambridge: Cambridge University Press, 2010. 
[3] Forsythe, N. et al., Application of a stochastic weather generator to assess climate change impacts in a semi-arid climate: The Upper Indus Basin. Journal of Hydrology, 517, pp. 1019-1034, 2014, ISSN 0022-1694.

[4] Fowler, H.J., Kilsby, C.G., O'Connell, P.E. \& Burton, A., A weather-type conditioned multi-site stochastic rainfall model for the generation of scenarios of climatic variability and change. J. Hydrol., 308(1-4), pp. 50-66, 2005.

[5] Wilby, R.L. \& Fowler, H.J., Regional Climate Downscaling: Modelling the Impact of Climate Change on Water Resources eds F. Fung, A. Lopez \& M. New, Blackwell Publishing Ltd, 2011. ISBN: 978-1-405-19671-0.

[6] Brown, C., Ghile, Y., Laverty, M. \& Li, K., Decision scaling: Linking bottom up vulnerability analysis with climate projections in the water sector. Water Resources Research, 48(9), 2012.

[7] Environment Canada, National climate data and information archive: Climate normals from 1971-2000, Environment Canada, 2012.

[8] Chen, J. \& Brissette, F., Comparison of five stochastic weather generators in simulating daily precipitation and temperature for the Loess Plateau of China. Intl. J. Climatol. 34(10), pp. 3089-3105, 2014.

[9] Baigorria, G.A. \& Jones, J.W., GiST: A stochastic model for generating spatially and temporally correlated daily rainfall data. J. Climate, 23(22), pp. 5990-6008, 2010.

[10] Wilks, D.S., Multi-site generalization of a daily stochastic precipitation model. $J$. Hydrol., 210, pp. 178-191, 1998.

[11] Wilby, R.W., Tomlinson, O.J. \& Dawson, C.W., Multisite simulation of precipitation by conditional resampling. Climate Res., 23(3), pp. 183-194, 2003.

[12] Palutikof, J.P., Goodess, C.M., Watkins, S.J. \& Holt, T., Generating rainfall and temperature scenarios at multiple sites: Examples from the Mediterranean. J. Climate, 15(24), pp. 3529-3548, 2002.

[13] Chen, J., Brissette, F. \& Zhang, X.J., A multi-site stochastic weather generator for daily precipitation and temperature. Transactions of the ASABE Trans. ASABE, 2014.

[14] Thom, H.C.S., A frequency distribution for precipitation. Bull. Amer. Meteorol. Soc., 32(10), p. 397, 1951.

[15] Buishand, T.A., Some remarks on the use of daily rainfall models. J. Hydrol., 36, pp. 295-308, 1978.

[16] Geng, S., Penning de Vries, F.W.T. \& Supit, I., A simple method for generating daily rainfall data. Agric. For. Meteorol., 36, pp. 363-376, 1986.

[17] Sen, Z. \& Eljadid, A.G., Rainfall distribution functions for Libya and Rainfall Prediction. Hydrol. Sci. J., 4(5), pp. 665-680, 1999.

[18] Woolhiser, D.A. \& Roldan, J., Stochastic daily precipitation models: 2. A comparison of distribution amounts. Water Resour. Res., 18(5), pp. 1461-1468, 1982.

[19] Burgueño, A., Martínez, M.D., Lana, X. \& Serra, C., Statistical distributions of the daily rainfall regime in Catalonia (Northeastern Spain) for the years 1950-2000. Int. J. Climatol., 25, pp. 1381-1403, 2005.

[20] Hanson, L.S. \& Vogel, R., The Probability Distribution of Daily Rainfall in the United States. Conference proceeding paper. World Environmental and Water Resources Congress, 2008.

[21] Chen, J., Brissette, F.P., Leconte, R. \& Caron, A., A versatile weather generator for daily precipitation and temperature. Trans. ASABE, 55(3), pp. 895-906, 2012.

[22] Richardson, C.W. \& Wright, D.A., WGEN: A Model for Generating Daily Weather Variables. U. S. Department of Agriculture. Agricultural Research Service, ARS-8, p. 83, 1984 . 
[23] Mehrotra, R., Srikanthan, R. \& Sharma, A., A comparison of three stochastic multisite precipitation occurrence generators. J. Hydrol., 331(1-2), pp. 280-292, 2006.

[24] Brissette, F.P., Khalili, M. \& Leconte, R., Efficient stochastic generation of multi-site synthetic precipitation data. J. Hydrol., 345(3-4), pp. 121-133, 2007.

[25] Goyal, M.K., Burn, D.H. \& Ojha, C.S.P., Precipitation simulation based on k-nearest neighbor approach using gamma kernel. J Hydrol Eng., 18, pp. 481-487, 2013.

[26] Sharif, M. \& Burn, D.H., Improved K-nearest neighbor weather generating model. $J$ Hydrol Eng., 12(1), pp. 42-51, 2007.

[27] Neitsch, S.L., Arnold, J.G., Kiniry, J.R. \& Williams, J.R., Soil and Water Assessment Tool Theoretical Documentation Version 2011, Grassland, Soil and Water Research Laboratory, Blackland Research Center, Temple, Texas, 2011.

[28] Srinivasan, R. \& Arnold, J.G., Integration of a basin-scale water quality model with GIS. Water Resour. Bull., 30(3), pp. 453-462, 1994.

[29] White, K.L. \& Chaubey, I., Sensitivity analysis, calibration, and validations for a multisite and multivariable SWAT model. J. American Water Resour. Assoc., 41(5), pp. 1077-1089, 2005.

[30] Tuppad, P., Douglas-Mankin, K.R., Lee, T., Srinivasan, R. \& Arnold, J.G., Soil and Water Assessment Tool (SWAT) hydrologic/water quality model: Extended capability and wider adoption. Trans. ASABE, 54(5), pp. 1677-1684, 2011.

[31] Arnold, J.G. et al., SWAT: Model use, calibration, and validation. Transactions of the ASABE, 55, pp. 1491-1508, 2012.

[32] Abbaspour, K.C., Vejdani, M. \& Haghighat, S., SWATCUP calibration and uncertainty programs for SWAT. In Proc. Intl. Congress on Modelling and Simulation (MODSIM'07), 1603-1609. L. Oxley \& D. Kulasiri, eds, Modelling and Simulation Society of Australia and New Zealand: Melbourne, Australia, 2007.

[33] Huber, P.J. \& Ronchetti, E.M., Robust Tests, in Robust Statistics, Second Edition, John Wiley \& Sons, Inc.: Hoboken, NJ, USA, 2009. doi: 10.1002/9780470434697.

[34] Fritsch, V., Varoquaux, G., Thyreau, B., Poline, J. \& Thirion, B., Detecting Outliers in High-Dimensional Neuroimaging Datasets with Robust Covariance Estimators. Medical Image Analysis, Elsevier, 16, pp. 1359-1370, 2012.

[35] Wang, M. \& Zwilling, C., Multivariate Computing and Robust Estimating for Outlier and Novelty in Data and Imaging Sciences, InTech, 2014.

[36] Hardin, J. \& Rocke, D.M., The distribution of robust distances. Journal of Computational and Graphical Statistics, 14, pp. 928-946, 2005.

[37] Liew, M.W., Veith, T.L., Bosch, D.D. \& Arnold, J.G., Suitability of SWAT for the conservation effects assessment project: A comparison on USDA-ARS experimental watersheds. J. Hydrologic Eng., 12(2), pp. 173-189, 2007.

[38] Moriasi, D.N. et al., Model evaluation guidelines for systematic quantification of accuracy in watershed simulations. ASABE, 50, p.3, 2007. 885í900.

[39] Lennartsson, J., Baxevani, A. \& Chen, D., Modelling precipitation in Sweden using multiple step Markov chains and a composite model. Journal of Hydrology, 363(1), pp. 42-59, 2008.

[40] Allard, D., Ailliot, P., Monbet, V. \& Naveau, P., Stochastic weather generators: An overview of weather type models. Le Journal de la Societ Francaise de Statistique, 156(1), pp. 101-113, 2015. 